\title{
ANÁLISE DA PROFILAXIA ANTIMICROBIANA PARA A PREVENÇÃO DA INFECÇÃO DO SÍTIO CIRÚRGICO EM UM HOSPITAL DO CENTRO-OESTE BRASILEIRO ${ }^{1}$
}

\author{
ANTIMICROBIAL PROPHYLAXIS ANALYSIS FOR PREVENTION OF \\ SURGICAL SITE INFECTION IN A BRAZIL CENTRE-WEST HOSPITAL
}

\author{
ANÁLISIS DE LA PROFILAXIS ANTIMICROBIANA PARA LA \\ PREVENCIÓN DE LA INFECCIÓN DEL SITIO QUIRÚRGICO EN UN \\ HOSPITAL DE LA REGIÓN CENTRO OESTE DE BRASIL
}

\author{
Cyanéa Ferreira Lima Gebrim ${ }^{*}$ \\ Jéssica Guimarães Rodrigues ${ }^{* *}$ \\ Maressa Noemia Rodrigues Queiroz ${ }^{* * *}$ \\ Regiane Aparecida Santos Soares Barreto ${ }^{* * * *}$ \\ Marinésia Aparecida Prado Palos ${ }^{* * * * *}$
}

\begin{abstract}
RESUMO
Este estudo objetiva analisar a profilaxia antimicrobiana no perioperatório de cirurgias limpas, em um hospital universitário do Centro-Oeste brasileiro. Estudo transversal descritivo, realizado em 700 prontuários de pacientes maiores ou igual a 18 anos, submetidos a procedimento cirúrgico limpo entre 2008 a 2010. Utilizou-se formulário estruturado e previamente avaliado. Para análise dos dados foram computados dois indicadores: a profilaxia antimicrobiana em até uma hora antes da incisão cirúrgica e a profilaxia antimicrobiana até 24 horas no pós-operatório, além das variáveis: antimicrobiano de escolha; dose de acordo com o peso do paciente e doses adicionais (repique) no intraoperatório, em cirurgias com tempo superior a 4 horas. Foram considerados os registros de até 30 dias após o procedimento cirúrgico ou de 12 meses nos casos de implantes de próteses e similares. Verificou-se que $86,6 \%$ receberam profilaxia antimicrobiana, em $75,1 \%$, a primeira dose obedeceu ao tempo preconizado e em 96,9\%, o antimicrobiano de escolha foi a Cefazolina. Houve inadequação em 70,6\% quanto à duração da profilaxia, $96,8 \%$ dose de acordo com o peso e $70 \%$ das doses adicionais. A taxa de infecção do sítio cirúrgico foi de 10\%, sendo o Staphylococcus aureus resistente à Meticilina, o agente etiológico mais frequente. Apesar das diretrizes referirem cautela quanto à profilaxia antimicrobiana, o estudo mostrou inadequações que podem trazer prejuízos para a segurança dos pacientes.
\end{abstract}

Palavras chave: Indicadores de qualidade em assistência à saúde, segurança do paciente, antibioticoprofilaxia, infecção da ferida operatória, enfermagem perioperatória.

\footnotetext{
${ }^{1}$ Extraído de dissertação de mestrado. Faculdade de Enfermagem/UFG; 2013.

* Enfermeira do Hospital das Clínicas da Universidade Federal de Goiás. Mestre em Enfermagem. Goiânia-Goiás. Brasil. Autor correspondente. E-mail: cyanealima@gmail.com

${ }^{* *}$ Estudante de Graduação em Enfermagem na Universidade Federal de Goiás. Goiânia-Goiás. Brasil. E-mail: jessicag.r@ hotmail.com

${ }^{* * *}$ Estudante de Graduação em Enfermagem na Universidade Federal de Goiás. Goiânia-Goiás. Brasil. E-mail: maressaqueiroz@hotmail.com

${ }^{* * * *}$ Enfermeira. Doutora em Enfermagem. Professora Adjunta da Faculdade de Enfermagem da Universidade Federal de Goiás. Goiânia-Goiás. Brasil. E-mail: remajuau@yahoo.com.br

${ }_{* * * * *}$ Enfermeira. Doutora em Enfermagem. Professora Adjunta da Faculdade de Enfermagem da Universidade Federal de Goiás. Goiânia-Goiás. Brasil. E-mail: marinesiaprado@gmail.com
} 


\begin{abstract}
The purpose of this study was to analyze preoperative antimicrobial prophylaxis in clean surgery, at a university hospital in Centre-West Brazil. This cross-sectional, descriptive study was completed using 700 records of patients 18 years of age or older undergoing clean surgery between 2008 and 2010. The study used a previously evaluated, structured form for data collection. Two indicators were analyzed: antimicrobial prophylaxis within one hour of incision, and antimicrobial prophylaxis 24 hours postoperatively, considering the following variables: antimicrobial selected; dose according to the weight of the patient, additional doses during surgery, and surgical procedures taking longer than four hours. Records completed within 30 days of the surgical procedure were considered, 12 months in cases of prosthetic implants and like ones. It was found that $86.6 \%$ had received antimicrobial prophylaxis, in $75.1 \%$, the first dose was administered within the recommended timeframe, and in $96.9 \%$, the antibiotic of choice was Cefazolin. Inadequacies were present in $70.6 \%$ for duration of prophylaxis, $96.8 \%$ in the dose according to the weight, and $70 \%$ in additional doses. The rate of surgical site infection was $10 \%$, methicillin resistant Staphylococcus aureus was the most common etiologic agent. Despite guidelines that advise caution with regard to antimicrobial prophylaxis, the study found inadequacies that may negatively impact patient safety.
\end{abstract}

Key words: Quality indicators, health care, patient safety, antibiotic prophylaxis, surgical wound infection, perioperative nursing.

\title{
RESUMEN
}

Este estudio tuvo como objetivo analizar la profilaxis antimicrobiana en el perioperatorio de cirugías limpias en un hospital clínico de la región Centro-Oeste de Brasil. Estudio transversal descriptivo, realizado en 700 historiales de pacientes mayores de 18 años, sometidos a procedimientos quirúrgicos limpios entre 2008 y 2010 . Se utilizó un formulario estructurado y previamente validado. Para analizar los datos fueron calculados dos indicadores: profilaxis antimicrobiana hasta antes de una hora de la incisión quirúrgica, y profilaxis antimicrobiana hasta 24 horas del postoperatorio, además de las siguientes variables: antibiótico elegido; dosis de acuerdo con el peso del paciente; dosis adicionales en el intraoperatorio, en cirugías de duración mayor a 4 horas. Fueron considerados los registros de hasta 30 días después del procedimiento quirúrgico, o 12 meses en los casos de implantes de prótesis o similares. Se verificó que el $86,6 \%$ de los pacientes recibió profilaxis antimicrobiana; en el 75,1\% de los pacientes la primera dosis obedeció al tiempo recomendado; y en el 96,9\% de los casos, el antibiótico elegido fue Cefazolina. Hubo disconformidades en el 70,6\% de los pacientes en cuando a la duración de la profilaxis, en el 96,8\% respecto a la dosis de acuerdo con el peso y en el 70\% de las dosis adicionales. La tasa de infección en el sitio quirúrgico fue de 10\%, siendo el Staphylococcus aureus resistente a la Meticilina, el agente etiológico más frecuente. A pesar de que las directrices indican cuidado en cuanto a la profilaxis antimicrobiana, el estudio mostró disconformidades que pueden provocar perjuicios en la seguridad de los pacientes.

Palabras clave: Indicadores de calidad de la atención de salud, seguridad del paciente, profilaxis antibiótica, infección de herida operatoria, enfermería perioperatoria.

Fecha recepción: 22/07/13. Fecha aceptación: 06/02/14.

\section{INTRODUÇÃO}

A preocupação com a segurança na assistência à saúde vem ascendendo desde 2004, ano em que a Organização Mundial da Saúde (OMS) lançou a Aliança Mundial para a Segurança do Paciente e Desafios Globais para a segurança no mundo todo. No perioperatório, período desde a decisão cirúrgica do paciente até o seu restabelecimento total, o programa "Cirurgias Seguras Salvam Vidas", proposto pela Aliança visa o comprometimento das equipes em adotar métodos para minimizar os riscos e eventos adversos (EA) provenientes da assistência (1). 
A OMS define EA, como uma ocorrência não intencional que causa dano ao paciente e pode resultar em incapacidade, disfunção temporária ou permanente, prolongamento de permanência hospitalar e até óbito como consequência do cuidado prestado (1).

Dentre os EA mais importantes, incluise as Infecções Relacionadas à Assistência à Saúde (IrAS) que acometem cerca de 1,7 milhão de pessoas no mundo, causando em torno de 100.000 mortes anualmente. Tratamse de infecções adquiridas pelos pacientes em consequência de cuidados e procedimentos de saúde, desde a atenção básica à alta complexidade e reabilitação. É a principal causa de morbimortalidade entre os pacientes hospitalizados, resultando em custos diretos e indiretos incalculáveis ao paciente, como o afastamento do convívio familiar e atividade profissional, durante o tratamento $(1,2)$.

Dentre as IrAS, sobressaem as infecções de sítio cirúrgico (ISC) que segundo estudo recente representam 31\%. Estima-se que somente nos Estados Unidos, 16 milhões de pacientes são submetidos a procedimentos cirúrgicos por ano, sendo que cerca de 300.000 a 500.000 adquirem esse agravo, representando cerca de 2 a $5 \%$ de ISC por ano, entretanto, estima-se que 40 a $60 \%$ dessas ocorrências sejam evitáveis (3-6).

Conceitua-se ISC, como um EA, no qual por algum motivo o micro-organismo penetra, se estabelece e multiplica na incisão operatória, acometendo tecidos, órgãos incisionados e cavidades manuseadas durante o procedimento cirúrgico (4). Pode ocorrer até 30 dias após o procedimento cirúrgico, mas em caso de implante de prótese ou similar, a vigilância se estende por até um ano $(4,7)$. É uma causa importante de morbidade, hospitalização prolongada, de 4 a 7 dias, aumenta duas vezes a chance do paciente necessitar de UTI, ir a óbito e cinco vezes mais risco de ser readmitido. Está associada a uma taxa de mortalidade de $3 \%$, sendo que $75 \%$ dos óbitos são diretamente atribuíveis a ela $(3,5,6)$.
Visando à segurança do paciente cirúrgico, a ISC agrega uma importante parcela do programa "Cirurgias Seguras Salvam Vidas" da OMS (7). Apoiando essa proposta, a Agência Nacional de Vigilância Sanitária (ANVISA) do Brasil, publicou o documento: "SÍTIO CIRÚRGICO - Critérios Nacionais de Infecções relacionadas à assistência à saúde" (8), no qual estabelece nove indicadores de processo para prevenção deste evento, a ser aplicado nas instituições brasileiras: 1) o tempo de internação pré-operatória menor ou igual a 24 horas em cirurgias eletivas; 2) a tricotomia com intervalo menor ou igual a duas horas; 3 ) o uso de tricotomizador elétrico ou tesoura; 4) a antissepsia do campo operatório; 5) o controle térmico nas cirurgias colorretais; 6) o controle glicêmico no pós-operatório imediato nas cirurgias cardíacas; 7) os registros de inspeção das caixas de materiais cirúrgicos, 8) a profilaxia antimicrobiana em até uma hora antes da incisão e 9) a profilaxia antimicrobiana até 24 horas no pós-operatório.

$\mathrm{O}$ uso da Profilaxia antimicrobiana (PATM), indicadores de número 8 e 9 da ANVISA, referem-se à administração de antibióticos de curto prazo nos pacientes antes do início das cirurgias. São relevantes para a prevenção e redução da incidência de ISC, desde que o uso do antimicrobiano (ATM) esteja em conformidade com as diretrizes, para evitar a seleção de cepas resistentes. Essa conduta diminui custos e reduz a morbimortalidade de pacientes associada às infecções (9). Sua indicação depende da classificação da ferida operatória, do tipo de cirurgia e das condições do paciente. Logo, deverá ser avaliada a sua vulnerabilidade a esses eventos, entre outros aspectos (10).

Usualmente, não se recomenda a PATM a pacientes submetidos às cirurgias limpas, pois a taxa de infecção da ferida cirúrgica não se modifica (11). Entretanto, essa conduta tem sido adotada nos casos de implante de prótese, enxerto ou eventual infecção, além das cirurgias cardíacas, e em pacientes de 
risco, como idosos, imunocomprometidos e portadores de diabetes mellitus $(7,10)$.

Sabe-se que a eficácia da PATM é alcançada quando administrada por via intravenosa, uma hora antes da incisão da pele, com o objetivo de atingir os níveis sanguíneos terapêuticos durante o transoperatório. Esse tempo é necessário para o ajustamento do nível tissular do ATM no momento da incisão, visando inibir ou eliminar as bactérias colonizantes da ferida cirúrgica ou do órgão abordado $(4,7,10)$.

O uso irracional dessa terapêutica na prática médica é um fato conhecido e que pode acarretar riscos à segurança dos pacientes. A importância da padronização de ATM é relevante em relação à economia para o hospital, e sua correta administração diminui a incidência de resistência bacteriana, a qual contribui para a diminuição das IrAS, bem como a redução do tempo de internação e custos hospitalares (9).

Nessa perspectiva, essa pesquisa vem fortalecer uma discussão necessária e pertinente na área da saúde, em especial à intervenção cirúrgica, no que se refere ao uso criterioso dos antimicrobianos. Trata-se de um tema atual e relevante pautado por evidências científicas.

Partindo desse princípio, esse estudo objetivou analisar a profilaxia antimicrobiana no perioperatório de cirurgias limpas em um hospital universitário da região Centro-Oeste do Brasil.

\section{MATERIAL E MÉTODO}

Estudo transversal descritivo, realizado em fonte de dados secundários, utilizando-se os registros de 3.823 prontuários de pacientes adultos, maiores ou igual a 18 anos, subme- tidos à cirurgia limpa, de janeiro de 2008 a dezembro de 2010, em um hospital universitário da região Centro-Oeste do Brasil.

Os prontuários foram obtidos por meio de classificação das cirurgias, junto à secretaria do Centro Cirúrgico e Núcleo Interno de Regulação do hospital do estudo. O projeto que contempla este estudo previu o levantamento em adultos, pelo fato de que a infecção em crianças merece discussões por especialistas em pediatria.

A amostra foi calculada em 626 prontuários, considerando a precisão de $2,5 \%$, para efeito de desenho de 1,5 e o intervalo de confiança de 95\%, aplicados à pesquisa. Em função das possíveis perdas, calculou-se acréscimo de 11,8\% ao tamanho da amostra, totalizando 700 prontuários.

A coleta de dados foi realizada por meio de busca ativa a partir dos registros dos procedimentos cirúrgicos nos prontuários. Ocorreu de julho a outubro de 2012, no Serviço de Arquivo Médico e Informação em Saúde (SAMIS) da referida instituição. Os dados foram registrados em um formulário estruturado, trazendo como eixo norteador, a definição de ISC e a PATM, variável prevista pela ANVISA com o um indicador (8).

As variáveis analisadas como fatores de risco para ISC foram: sexo, idade, classificação do estado físico de acordo com a escala da American Society of Anaesthesiologists (ASA), doença crônica, peso, desnutrição, alergia, etilismo e tabagismo, implante de prótese ou similar, instalação de dreno e duração da cirurgia (3-5).

Para análise da PATM, foram computados os dois indicadores propostos pela ANVISA (8): a profilaxia antimicrobiana em até uma hora antes da incisão e a profilaxia antimicrobiana até 24 horas no pós-operatório, ambos calculados a partir de duas fórmulas, respectivamente: 


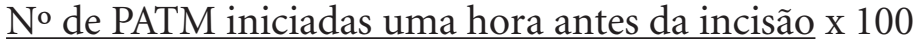 Número de PATM avaliadas}

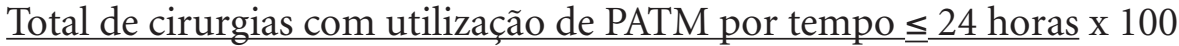

Total de cirurgias com utilização de PATM

De forma sistemática, averiguou-se toda prescrição de antimicrobiano profilático e foram eleitas, além dos dois indicadores descritos anteriormente, as seguintes variáveis relacionadas à PATM: antimicrobiano de escolha; dose de acordo com o peso do paciente e doses adicionais (repique) no intraoperatório, em cirurgias com tempo superior a 4 horas.

Foram considerados os registros de PATM nos prontuários no período de até 30 dias após o procedimento cirúrgico ou de 12 meses nos casos de implantes de próteses e similares.

As informações foram analisadas de forma descritiva e os resultados foram dispostos em frequência absoluta e porcentagem por meio do programa Statistical Package for the Social Science (SPSS) versão 15.0.

Atentou-se para os aspectos éticos e legais de acordo com a Resolução 466/2012 do Ministério da Saúde do Brasil (12). O projeto teve a aprovação do $\mathrm{CEP} / \mathrm{HC} / \mathrm{UFG}$ No 06/2012, No CAAE Plataforma Brasil: 02052912.0.0000.5078.

\section{RESULTADOS}

Foram revisados 700 prontuários de pacientes submetidos à cirurgia limpa. A maioria $(57,1 \%)$ do sexo feminino, com idade entre 18 a 101 anos e predominância da faixa etária entre 18 e 59 anos (70,0\%) (Tabela 1 ).

As variáveis analisadas foram os fatores de risco para ISC, sendo que a PATM se relaciona com a prevenção da infecção dessa topografia.

Quanto à gravidade dos pacientes, de acordo com a Americam Society of Anesthesiology (ASA) a maioria $(74,6 \%)$ recebeu a classificação ASA I (Pacientes saudáveis) e II (Doença sistêmica leve ou moderada). A condição de portador de doenças crônicas, como Diabetes Mellitus (DM), hipertensão arterial (HAS), insuficiência renal (IRC) e obesidade foram observadas em 275 (39,3\%) casos. E, 83 (11,9\%) pacientes exibiam diagnóstico de infecção em outro sítio (à distância) (Tabela 1).

Tabela 1. Caracterização dos pacientes, submetidos a cirurgias limpas, em um hospital universitário, no período de 2008 a 2010, segundo os fatores de risco para infecção do sítio cirúrgico. Goiânia, 2012.

\begin{tabular}{llcc}
\hline \multicolumn{2}{l}{ Variáveis } & $\mathrm{N}$ & $\%$ \\
\hline Sexo & & & \\
& Masculino & 300 & 42,9 \\
& Feminino & 400 & 57,1 \\
Idade & & & \\
& $18-29$ & 139 & 19,9 \\
& $30-49$ & 244 & 34,9 \\
& $50-59$ & 106 & 15,1 \\
& $60-69$ & 108 & 15,4 \\
& $70-101$ & 103 & 14,7 \\
\hline
\end{tabular}


Continuação Tabela 1.

\begin{tabular}{|c|c|c|}
\hline \multicolumn{3}{|l|}{ Tipo de ASA } \\
\hline $\mathrm{ASA} \leq \mathrm{II}$ & 522 & 74,6 \\
\hline $\mathrm{ASA} \geq \mathrm{III}$ & 178 & 25,4 \\
\hline \multicolumn{3}{|c|}{ Doença Crônica (HAS, DM, IRC e Obesidade) } \\
\hline Sim & 275 & 39,3 \\
\hline Não & 425 & 60,7 \\
\hline \multicolumn{3}{|l|}{ Desnutrição } \\
\hline Sim & 9 & 1,3 \\
\hline Não & 512 & 73,1 \\
\hline Sem registro & 179 & 25,6 \\
\hline \multicolumn{3}{|l|}{ Peso } \\
\hline$\leq 80 \mathrm{~kg}$ & 427 & 61,0 \\
\hline$>80 \mathrm{~kg}$ & 70 & 10,0 \\
\hline Sem registro & 203 & 29,0 \\
\hline \multicolumn{3}{|l|}{ Alergia à Cefalosporina } \\
\hline Sim & 2 & 0,3 \\
\hline Sem registro & 698 & 99,7 \\
\hline \multicolumn{3}{|c|}{ Uso de Prótese ou similares } \\
\hline Sim & 324 & 46,3 \\
\hline Não & 376 & 53,7 \\
\hline \multicolumn{3}{|l|}{ Uso de dreno } \\
\hline Sim & 228 & 32,6 \\
\hline Não & 472 & 67,4 \\
\hline \multicolumn{3}{|l|}{ Duração da Cirurgia } \\
\hline$\leq 4$ horas & 580 & 82,9 \\
\hline$>4$ horas & 120 & 17,1 \\
\hline \multicolumn{3}{|l|}{ Imunossupressão } \\
\hline Sim & 47 & 6,7 \\
\hline Não & 642 & 91,7 \\
\hline Sem registro & 11 & 1,6 \\
\hline \multicolumn{3}{|c|}{ Infecção à distância do sítio cirúrgico } \\
\hline Sim & 83 & 11,9 \\
\hline Não & 617 & 88,1 \\
\hline \multicolumn{3}{|l|}{ Etilismo } \\
\hline Sim & 55 & 7,9 \\
\hline Não & 354 & 50,6 \\
\hline Ex-etilismo & 08 & 1,1 \\
\hline Sem registro & 283 & 40,4 \\
\hline \multicolumn{3}{|l|}{ Tabagismo } \\
\hline Sim & 170 & 24,3 \\
\hline Não & 344 & 49,1 \\
\hline Ex-tabagismo & 31 & 4,5 \\
\hline Sem registro & 155 & 22,1 \\
\hline \multicolumn{3}{|c|}{ Infecção do Sítio Cirúrgico } \\
\hline Sim & 70 & 10,0 \\
\hline Não & 630 & 90,0 \\
\hline Total & 700 & 100 \\
\hline
\end{tabular}

O tabagismo e o etilismo foram observados em $170(24,3 \%)$ e $55(7,9 \%)$ pacientes, respectivamente. A falta do registro desses dois fatores de risco foi representativa, 155 $(22,1 \%)$ e $283(40,4 \%)$ dos casos, respectiva- mente. Já em relação ao peso dos pacientes, 427 (61\%) apresentavam até $80 \mathrm{~kg}, 70$ (10\%) pacientes apresentaram peso superior a esse e, em 203 (29\%), não havia registro de peso no prontuário (Tabela 1 ). 
Considerando os indicadores de processo para prevenção de ISC previstos pela ANVISA, a PATM foi realizada em 606 pacientes, $86,6 \%$ da amostra. Desse total, o tempo de administração da primeira dose do antimi- crobiano, em $455(75,1 \%)$, ocorreu conforme o preconizado, até uma hora antes da incisão da pele. Mas, $112(18,5 \%)$ não seguiram esse parâmetro e em $39(6,4 \%)$ essas informações não foram registradas (Gráfico 1).

Gráfico 1. Profilaxia antimicrobiana no intraoperatório de cirurgias limpas, no período de 2008 a 2010, segundo as variáveis analisadas. Goiânia, 2012.

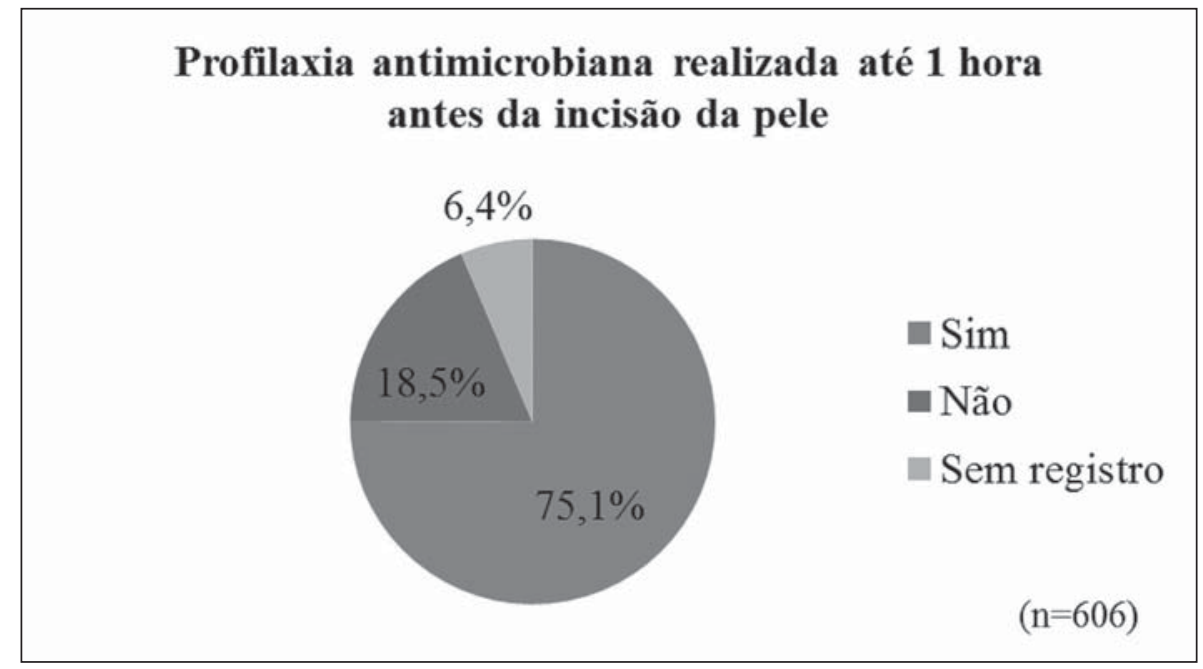

A Cefazolina foi o ATM de escolha em 587 $(96,9 \%)$ casos. Havia dois $(0,3)$ registros de pacientes que referiram ser alérgicos à Cefalosporina. Destaca-se que informações sobre alergias não foram encontradas em 99,7\% dos prontuários.

Considerando a Cefazolina como a dro- ga de escolha pela equipe médica, 17 (2,9\%) pacientes receberam $1 \mathrm{~g}$ desse ATM e 570 $(97,1 \%)$ pacientes receberam $2 \mathrm{~g}$. Em relação à dose/peso, um (1,7\%) paciente recebeu subdose, em contrapartida, em 360 (96,8\%), foram administradas doses acima da recomendada (Tabela 2).

Tabela 2. Distribuição da dose de Cefazolina em procedimento cirúrgico limpo, segundo o peso do paciente, em um hospital universitário, no período de 2008 a 2010. Goiânia, 2012.

\begin{tabular}{lccccc}
\hline & & & Peso & & Total \\
& & & & & \\
& & & & \\
& & $80 \mathrm{~kg}$ & Sem registro & $\mathrm{N}(\%)$ & $\mathrm{N}(\%)$ \\
\hline \multirow{2}{*}{ Doses } & $1 \mathrm{~g}$ & $12(3,2)$ & $1(1,7)$ & $4(2,7)$ & $17(2,9)$ \\
& $2 \mathrm{~g}$ & $360(96,8)$ & $58(98,3)$ & $152(97,3)$ & $570(97,1)$ \\
\hline \multicolumn{2}{c}{ Total } & 372 & 59 & 156 & 587 \\
\hline
\end{tabular}


Em relação ao tempo da cirurgia, 580 $(82,9 \%)$ ocorreram em um período de até 4 horas de duração. Observou-se que, em 120 $(17,1 \%)$, o tempo de duração foi superior a 4 horas. Dessas, 84 (70\%) pacientes não receberam doses adicionais (repique) da Cefazolina e apenas $36(30 \%)$ receberam o repique conforme preconizado pela legislação $(7,8)$.

Quanto à duração da PATM cirúrgica, 494 (70,6\%) excederam às 24 horas e, apenas, 206 $(29,4 \%)$ receberam o ATM por até 24 horas de acordo com o recomendado $(7,8)$.

Em decorrência do número elevado $(70,6 \%)$ de pacientes que receberam PATM por mais de 24 horas, procurou-se conhecer os aspectos que possivelmente determinaram essa conduta por parte da equipe médica.
Constatou-se que aos pacientes submetidos às cirurgias com implante de prótese e similares houve duração maior que 24 horas da administração de ATM, em 264 (53,4\%) casos, nas especialidades ortopedia $(39,7 \%)$, vascular $(21,5 \%)$ e plástica $(19,8 \%)$.

A incidência da ISC, nesse estudo, foi de $70(10 \%)$ eventos. Apenas $19(27,1 \%)$ constavam resultados de culturas, todas referentes a micro-organismos multirresistentes. Dentre esses, 26,1\%, Staphylococcus aureus resistente à Meticilina (MRSA), 17,4\%, Pseudomonas aeruginosa multirresistente e 13,0\%, Acinetobacter baumannii (Gráfico 2). Ressalta-se a presença de quatro pacientes que apresentaram infecções com dois germes ao mesmo tempo.

Gráfico 2. Micro-organismos isolados dos pacientes submetidos à cirurgia limpa, no período de 2008 a 2010, em um hospital universitário. Goiânia, 2012.

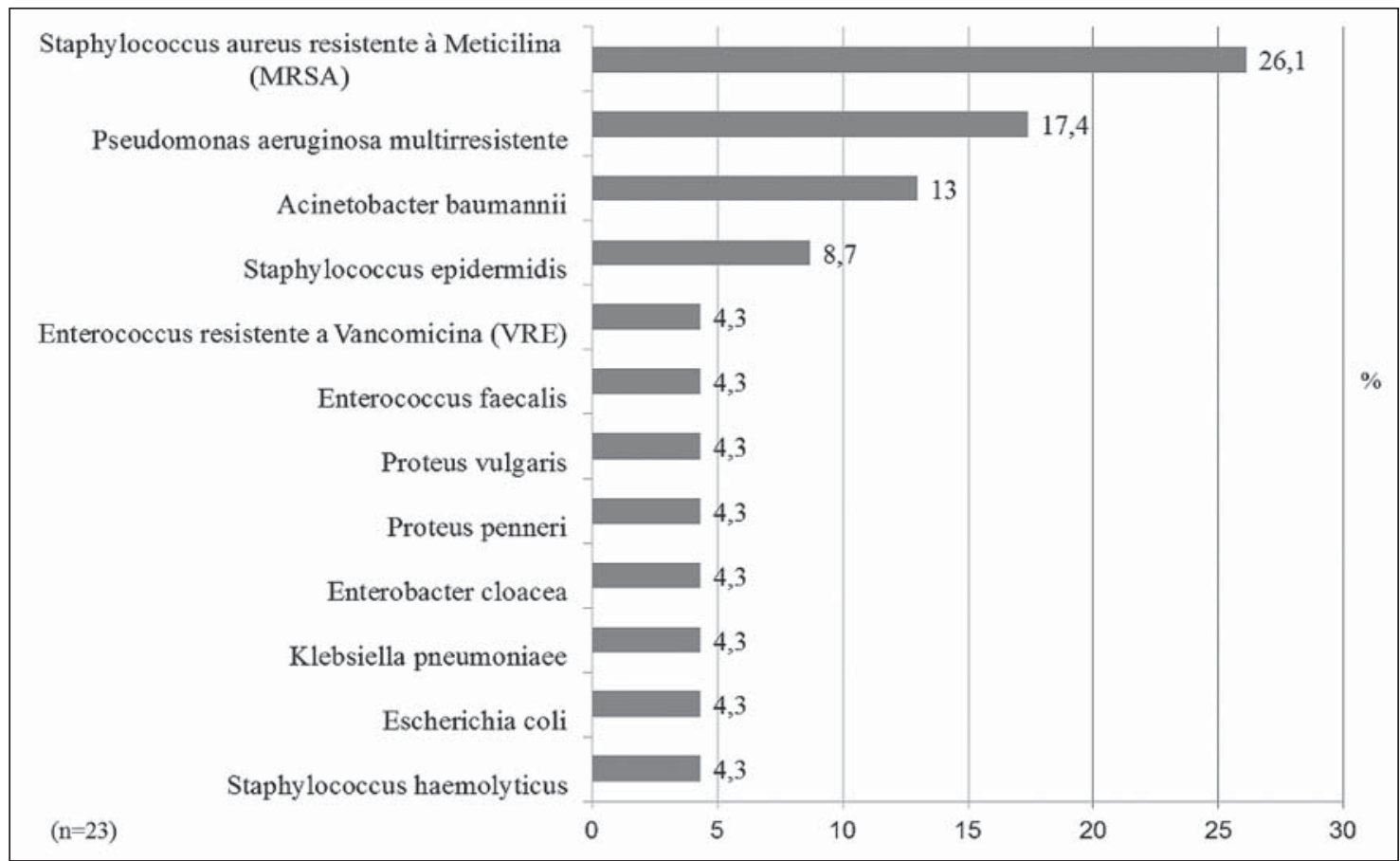




\section{DISCUSSÃO E CONCLUSÃO}

A profilaxia antimicrobiana cirúrgica (PATM), de acordo com as diretrizes, faz-se necessária com o intuito de diminuir a incidência de ISC, pois a ação de rompimento da barreira epitelial, durante a intervenção cirúrgica, desencadeia reações sistêmicas que promovem o processo infeccioso, seja ele no campo operatório, em procedimento invasivo distinto, ou em outro foco à distância (13).

Entretanto, a eficácia da PATM depende de aspectos imprescindíveis, como por exemplo, o momento de início da profilaxia, a necessidade de repetição no intraoperatório, bem como o tempo de duração $(10,14)$. Conforme o Treatment Guidelines from the Medical Letter (14), o tempo adequado para infusão da primeira dose do ATM é de até uma hora antes da incisão da pele. Essa medida confere a presença do ATM nos tecidos manuseados, no momento em que há a exposição do sítio cirúrgico aos micro-organismos, pois o período preconizado permite aos ATM atingirem níveis séricos e teciduais adequados $(4,13)$.

Evidências comprovaram que, quando o ATM é instituído incorretamente, sua eficácia pode ser comprometida, independente da dose ou duração do esquema $(3,10,15-17)$.

A escolha do ATM requer julgamento clínico assertivo e rigoroso critério de seleção, incluindo a microbiota da pele, o espectro de ação e a farmacocinética desses medicamentos. É necessário ainda atentar para outros aspectos como, menor toxicidade e indução de resistência, mecanismos de ação, limiar de concentração no sítio cirúrgico, posologia e via de administração eficaz $(8,13,14,16,18$ 20).

As cefalosporinas de primeira geração são as mais empregadas na PATM cirúrgica $(3,7$, $8,14,16,20,21)$. Lembrando que, a Cefazolina, por exemplo, liga-se em maior quantidade as proteínas plasmáticas, tem volume de distribuição mais baixo, clearance reduzido e meia vida de 1-2 horas. Esses fatores propor- cionam este medicamento a atingir concentrações séricas mais elevadas e ampliação do platô de ação, tornando-se o ATM de escolha em profilaxia cirúrgica. Vale lembrar que se trata de medicamento com risco alérgico ao paciente, portanto, recomenda-se como alternativa, a Clindamicina e a Vancomicina $(3,7,8,16,20)$.

Visando à eficácia da Cefazolina, as diretrizes indicam que esse ATM deve ser administrado conforme o peso do paciente. Em adultos com peso inferior a $80 \mathrm{~kg}$, a dose indicada é de $1 \mathrm{~g}$ e superior a $80 \mathrm{~kg}$ a dose é de $2 \mathrm{~g}(18,20,22)$.

O uso de dose extra do ATM, não confere benefício adicional à profilaxia e ainda resulta no aumento dos custos (9). Além disso, favorece a seleção de cepas bacterianas resistentes, a exemplo das descritas nesse estudo a Pseudomonas aeruginosa, Acinetobacter baumannii, Klebsiella pneumoniae e surgimento de novas betalactamases $(19,22)$.

No caso de pacientes obesos, faz-se necessário o ajustamento das doses, pois esses pacientes dispõem de uma massa corporal que absorve, metaboliza e excreta os ATM por mecanismos diferenciados. A obesidade ainda provoca uma alteração complexa dos parâmetros farmacocinéticos, em relação ao volume de distribuição e o clearance corporal total. Esse quesito exige um ajustamento de doses, visando a efetividade antimicrobiana (23). Recomenda-se a ampliação da dose para $2 \mathrm{~g}$ de Cefazolina, visando alcançar a cobertura profilática e evitar as complicações pós-cirúrgicas $(18,20,22)$.

O ajustamento da dose do ATM também é recomendado no caso de etilismo. A interação do álcool com fármacos advém da indução enzimática, nos casos de alcoolismo crônico, da inibição do metabolismo das drogas, em casos de intoxicação alcoólica aguda e da disfunção hepática grave causada pelo álcool (24). O álcool consiste ainda em um fator de risco para ISC, pois atua como imunossupressor, como por exemplo, a supressão do fator de necrose tumoral, essencial para a res- 
posta imune $(25,26)$.

Imediatamente ao ajuste da dose, faz-se necessária averiguar a precisão de dose adicional (repique) durante o procedimento cirúrgico. Levando em conta a meia-vida da Cefazolina, o que permite maior flexibilidade quanto ao horário da administração e que o tempo da administração da dose adicional seja de duas vezes a meia-vida do medicamento, recomenda-se que o repique seja realizado, dentro de quatro horas após a primeira dose, assegurando a manutenção dos níveis séricos e teciduais da droga. Essa recomendação quando não seguida pode acarretar a não eficácia da profilaxia, expondo o paciente a risco de colonização por bactéria resistente $(14,15,22,27)$.

Sistematicamente, estudos vêm mostrando a ausência de justificativa para o uso prolongado de ATM profilático, pois mesmo que haja eficácia, o potencial-benefício da continuidade do uso será certamente ofuscado pelo desenvolvimento de efeitos adversos, seleção de cepas bacterianas resistentes e falhas na terapêutica, além de aumentar custos para a instituição $(14-17,19,20)$.

Os achados dessa investigação corroboram com a ocorrência de infecção pós-operatória averiguada no estudo de Sérgio-Segundo et al. (28) em que comparando dois grupos de pacientes submetidos à cirurgia plástica estética, em uso de PATM, identificou que o uso correto de doses profiláticas de antibióticos não foi associado a episódios de infecção, em detrimento da terapêutica antimicrobiana prolongada que não impediu a ocorrência de infecção do sítio cirúrgico. Os autores alertam, ainda, para instituir criteriosamente a PATM como uma das peças importantes na prevenção dessa infecção.

Vários estudos advertem e não recomendam o uso prolongado da PATM, após as 24 horas da cirurgia, em casos de prótese ou similares, uma vez que a extensão da duração da profilaxia não é eficaz nessa situação e seria utilizada como tratamento de infecções e não mais em profilaxia $(7,10,14,18,29)$.
Em se tratando do índice de ISC aceitável pelo Centers for Disease Control (CDC), nos casos de cirurgias limpas é de até $5 \%(4,7,8)$. Esse índice não foi alcançado nesse estudo, pois a taxa de ISC foi o dobro da referenciada pelo CDC.

A profilaxia antimicrobiana deve seguir rigorosamente os critérios definidos para o uso desses medicamentos, visando dentre outros eventos, à seleção de cepas resistentes. Em se tratando de cepas resistentes e em especial as identificadas nos prontuários dos pacientes submetidos à intervenção cirúrgica limpa, destaca-se que uma única cepa resistente pode ser disseminada rapidamente em toda instituição, além da comunidade em geral (30).

A análise da profilaxia antimicrobiana no perioperatório de cirurgias limpas em um hospital universitário da região Centro-Oeste do Brasil, mostrou que dos indicadores previstos pela Agência Nacional de Vigilância Sanitária do Brasil, o tempo de administração da primeira dose do antimicrobiano até uma hora antes da incisão da pele foi considerado adequado; também foi considerada adequada a variável: antimicrobiano de escolha, sendo a Cefazolina a medicação eleita em quase $100 \%$ das profilaxias realizadas.

Em contrapartida, o indicador duração da profilaxia antimicrobiana cirúrgica de até 24 horas e as variáveis: dose de acordo com o peso do paciente e doses adicionais (repique) no intraoperatório, em cirurgias com tempo superior a 4 horas, todos foram considerados inadequados.

A taxa de infecção do sítio cirúrgico incidiu em 10\% das cirurgias limpas e evidenciou-se uma tendência ao uso excessivo de antimicrobiano. A resistência microbiana foi constatada em $100 \%$ dos resultados de culturas registrados nos prontuários, sendo o Staphylococcus aureus resistentes à Meticilina (MRSA), o micro-organismo de maior ocorrência.

Os dados da pesquisa demonstraram inadequação no uso da profilaxia antimicrobia- 
na, no serviço analisado, segundo as diretrizes do país. Essa medida deve ser revista, visando à segurança dos pacientes e trabalhadores do serviço, para a prevenção e controle da colonização e eventual infecção, em especial a infecção do sítio cirúrgico.

Esses achados reforçam que medidas de intervenção sejam aplicadas no serviço, como exemplo, colocar em prática uma política efetiva para a aquisição e vigilância do uso de antimicrobianos; concretizar a elaboração de protocolos norteados por indicadores estabelecidos pelas diretrizes nacionais e internacionais; envolver os serviços de cirurgia, anestesiologia, farmácia e comissão de controle de infecção e uniformizar as condutas, uma vez que a multirresistência é uma ameaça global à segurança do paciente.

Dentre as limitações do estudo, ressaltase a subnotificação dos dados nos prontuários, pois se tratou de estudo retrospectivo, de fonte documental, ou seja, dependente da acurácia dos registros realizados pelos profissionais de saúde.

Conflitos de interesse: não há conflitos de interesse a declarar.

\section{REFERÊNCIAS}

1. World Health Organization (WHO). World Alliance for Patient Safety: Forward Programme 2008-2009. Geneva: World Health Organization; 2008.

2. Siegel JD, Rhinehart E, Jackson M, Chiarello $\mathrm{L}$, and the Healthcare Infection Control Practices Advisory Committee. 2007. Guideline for Isolation Precautions: Preventing Transmission of Infectious Agents in Healthcare Settings. [Internet]. Atlanta: Centers for Disease Control and Prevention; 2007 [citado 2 agosto 2014]. Disponível em: http://www.cdc.gov/hicpac/pdf/isolation/Isolation2007.pdf

3. SIS-LA. Surgical Infection Society-Latin
America. Manual Latino-americano de Guias Baseadas na Evidência: Estratégias para a Prevenção da Infecção Associada ao Atendimento na Saúde-ESPIAAS. Bogotá: Gustavo A Quintero; 2009.

4. Associação Paulista de Estudos e Controle de Infecção Hospitalar (APECIH). Prevenção de infecção de sítio cirúrgico. 3 ed. São Paulo: APECIH; 2009.

5. Anderson DJ. Surgical Site Infections. En: Division of Infectious Diseases, editor. Durham: Elsevier; 2011.p. 135-53.

6. Centers for Disease Control and Prevention. Surgical Site Infection (SSI) Event. Procedure-associated Module.[Internet]. Atlanta: CDC; 2014 [citado 2 agosto 2014]. Disponível em: http://www.cdc. gov/nhsn/PDFs/pscManual/9pscSSIcurrent.pdf?agree $=$ yes\&next $=$ Accept

7. Organização Mundial da Saúde (OMS). Segundo desafio global para a segurança do paciente: Cirurgias seguras salvam vidas (orientações para cirurgia segura da OMS). [Internet]. Rio de Janeiro: Organização Pan-Americana da Saúde; Ministério da Saúde; Agência Nacional de Vigilância Sanitária; 2009 [citado em 2 agosto 2014]. Disponível em: http://bvsms.saude.gov.br/bvs/publicacoes/seguranca_paciente_cirurgia_salva_manual. pdf

8. Agência Nacional de Vigilância Sanitária (ANVISA). Sítio cirúrgico: Critérios nacionais de infecções relacionadas à assistência à saúde. Brasília: Ministério da Saúde; 2009.

9. Mota LM, Vilar FC, Dias LBA, Nunes TF, Moriguti JC. Uso racional de antimicrobianos. Medicina (Ribeirão Preto). 2010; 43(2): 164-72.

10. Magalhães ACM, Rodney-Neto MN, Roesberg J, Hayck J, Silva AL, et al. Diretrizes Clínicas. Antibioticoprofilaxia Cirúrgica. Procedimento Operacional Padrão - Protocolo Clínico 028. Minas Gerais: FHEMIG; 2011.

11. Setiawan B. The role of prophylactic an- 
tibiotics in preventing perioperative infection. Acta Med Indones. 2011; 43(3): 262-6.

12. Ministério da Saúde, Conselho Nacional de Saúde. Resolução No 466/12 - Diretrizes e normas regulamentadoras para pesquisas envolvendo seres humanos. [Internet]. Brasília: Ministério da Saúde; 2012 [citado 2 agosto 2014]. Disponível em: http://bvsms.saude.gov.br/bvs/saudelegis/cns/2013/res0466_12_12_2012.html

13. Fanhani HR, Beltrão L. Uso inadequado das cefalosporinas e a atuação da Comissão de Controle de Infecção Hospitalar. Saúde e Biol. 2011; 6(3): 70-82.

14. Treatment Guidelines from The Medical Letter. Antimicrobial Prophylaxis for Surgery. The Medical Letter. 2009; 7(82): 47-52.

15. Steinberg JP, Braun BI, Hellinger WC, Kusek L, Bozikis MR, Bush AJ, et al. Timing of Antimicrobial Prophylaxis and the Risk of Surgical Site Infections: Results From the Trial to Reduce Antimicrobial Prophylaxis Errors. Ann Surg. 2009; 250(1): 10-6.

16. Scottish Intercollegiate Guidelines Network. Antibiotic prophylaxis in surgery: A national clinical guideline. Scotland: Elliott House; 2008.

17. Anderson DJ, Kaye KS, Classen D, Arias KM, Podgorny K, Burstin H, et al. Strategies to Prevent Surgical Site Infections in Acute Care Hospitals. Infect Control Hosp Epidemiol. 2008; 29 Suppl 1: S5161.

18. Croco EL, Nakagawa C. Uso de antibioticoprofilaxia em cirurgia. Rev Fac Ciênc Méd Sorocaba. 2008; 10(3): 30-7.

19. Santos RP, Nagel F, Gastal SL, Sander GB, Jacobi TS, Konkewicz LR, et al. Política de antimicrobianos do Hospital de Clínicas de Porto Alegre: 2010 Comissão de Controle de Infecção Hospitalar. Rev HCPA. 2010; 30(1): 13-21.

20. Setiawan B. The Role of Prophylactic Antibiotics in Preventing Perioperative In- fection. Acta Med Indones. 2011; 43(4): 262-6.

21. Guilarde AO, Pacheco IT, Gomes LVR, Lima VAB, Pacheco JP, Souza MA. Avaliação da antibioticoprofilaxia cirúrgica em hospital universitário. Rev de Patologia Tropical. 2009; 38(3): 179-85.

22. Pires MR, Gastal SL, Silva CF, Dallé J, Deutschendorf C, Kuplich NM, et al. Avaliação do uso de cefazolina como profilaxia antibiótica em procedimentos cirúrgicos. Rev HCPA. 2012; 32(1): 18-23.

23. Ho VP, Nicolau DP, Dakin GF, Pomp A, Rich BS, Towe CW, et al. Cefazolin dosing for surgical prophylaxis in morbidly obese patients. Surg Infect (Larchmt). 2012; 13(1): 33-7.

24. Broadwater M, Varlinskaya EI, Spear LP. Chronic intermittent ethanol exposure in early adolescent and adult male rats: effects on tolerance, social behavior, and ethanol intake. Alcohol Clin Exp Res. 2011; 35(8): 1392-403.

25. Daneman N, Lu H, Redelmeier DA. J Hosp Infect. Discharge after discharge: predicting surgical site infections after patients leave hospital. 2010; 75(3): 18894.

26. Neumayer L, Hosokawa P, Itani K, El-Tamer M, Henderson WG, Khuri SF. Multivariable predictors of postoperative surgical site infection after general and vascular surgery: results from the patient safety in surgery study. J Am Coll Surg. 2007; 204(6): 1178-87.

27. Adembri C, Ristori R, Chelazzi C, Arrigucci S, Cassetta MI, De-Gaudio AR, et al. Cefazolin bolus and continuous administration for elective cardiac surgery: improved pharmacokinetic and pharmacodynamic parameters. J Thorac Cardiovasc Surg. 2010; 140(2): 471-5.

28. Sérgio-Segundo SWGO, Paula PRS, Souza LHG, Prado M, Cavalcante LM, Sobrinho EFSS. Uso terapêutico vs. profilático de antibiótico em cirurgias plásticas estéticas: estudo retrospectivo/descritivo no 
Hospital das Clínicas da Universidade Federal de Goiás. Rev Bras Cir Plást. 2011; 26(supl): 12.

29. Lichtenfels E, Frankini AD, Cardozo MA, D'Azevedo PA. Infecção em endoprótese. J Vasc Bras 2011; 10(1): 50-4.
30. Oliveira AC, Silva RS. Desafios do cuidar em saúde frente à resistência bacteriana: uma revisão. Rev Eletr Enf [Internet]. 2008 [citado 29 ene 2014]; 10(1): 189-97. Disponível em: http://www.fen.ufg.br/revista/v10/n1/v10n1a17.htm 